Thomas E. Balke

\title{
Eine Fāra-zeitliche Urkunde aus dem Völkerkundemuseum in Werl
}

\begin{abstract}
The article presents a hitherto unpublished ED IIIa tablet with a two-column inscription on its obverse housed in the Ethnological Museum in Werl, Germany. Based on its physical features, contextual structure and prosopography it is in all likelihood datable to the Fāra-period and probably comes from the site of Fāra itself, ancient Šuruppak. In spite of its obvious brevity, the content proves to be quite intricate. It seems rather probable that the text should be considered as a kind of draft for a legal record possibly dealing with a matter of inheritance.
\end{abstract}

Keywords: Fāra, sale contract, Sumerian personal names, legal text

DOI 10.1515/aofo-2014-0003

Die hier vorzustellende Tontafel, die bislang unpubliziert geblieben ist, ist unter der Museums-Nr. MW 172d Bestandteil der umfangreichen Sammlung des Völkerkundemuseums „Forum der Völker“ der Franziskaner in Werl. Das Museum verdankt die Tontafel einer Schenkung des Soester Sammlers und CDU-Bundestagsabgeordneten Dr. Ernst Majonica (1920-1997). ${ }^{1}$ Die nur mit zwei Kolumnen beschriebene undatierte Tafel, die konkav nach außen gewölbt und aus bräunlich-gelbem Ton gefertigt ist, erweckt zunächst den Eindruck einer kurzen Notiz bzw. eines Entwurfes einer Feldkaufurkunde, wenngleich viele der maßgeblichen Elemente und Angaben Fāra-zeitlicher Rechtsurkunden fehlen wie der Kaufpreis in Kupfer ( $n$ uruda ma-na, sám-bé), ein Käufer (lú [aša ${ }_{5} /$ é] sa $\mathrm{s}_{10}$ ) und Verkäufer (lú sám $\mathrm{gu}_{7}$ ), das eigentliche Verkaufsformular (é e-šè-sa $a_{10}$ ) oder eine abschließende Zeugenliste (lú ki inim-ma) bzw. Datierung (bala PN). ${ }^{2}$ Lediglich der charakteristische Terminus ní $\hat{g}$-ba „Gabe, Geschenk“, der oftmals gemeinsam mit der Zusatzzahlung ní $\hat{g}$-diri(-g) im Formular Fāra-zeitlicher Kaufurkunden erscheint, ist in unserer Urkunde vertreten. Sieht man dies zusammen mit dem Tafelformat und dem Schriftduktus sowie den genannten, aus dem FāraOnomastikon hinreichend bekannten Personennamen Ur-lugal-gub(DU) und Ĝišgal-si, ist die Tafel sicher in die Fāra-Zeit oder möglicherweise etwas jünger zu datieren. Da Ersterer ausschließlich in Urkunden aus Šuruppak (Fāra) belegt ist und m.W. nicht in den als Fāra-zeitlich eingestuften Texten anderer Provenienz, z.B. aus Adab, Girsu, Nippur oder Uruk, ${ }^{3}$ stammt unsere Tafel möglicherweise tatsächlich aus Fāra, obwohl dies nicht zwingend ist. Der zwar knappe doch nicht leicht fassbare Text nennt 5 recht große Flurstücke mit nachfolgender Lokalisation oder Zuordnung zu einer Person, ${ }^{4}$ wenngleich sowohl einige der Toponyme als auch der Personennamen bislang in der Fāra-Zeit unbekannt sind; allerdings scheint die

\footnotetext{
1 Die Keilschrifttafel gehörte zur umfangreichen Sammlung von Dr. Ernst Majonica, der bereits Jahre vor seinem Tod 1997 sowohl diese Tafel als auch die umfangreiche völkerkundliche Sammlung mit ca. 1000 Exponaten dem Museum Forum der Völker in Werl testamentarisch vermacht hatte; aufgrund seines schlechten Gesundheitszustandes konnte das Museum bei der Sichtung der Sammlung in den 1990er-Jahren über die Herkunft und mögliche Provenienz der Tafel leider keine Informationen mehr erhalten. 2 Zur Struktur und Terminologie der Rechtsurkunden der Fāra-Zeit vgl. Wilcke (2007: 80-84), exemplarisch sei hier verwiesen auf die Urkunde T. Gomi, Orient 19 (1983) 2ff.

3 Vgl. die Liste bei Krebernik (1998: 372-377).

4 Wahrscheinlicher scheint mir, dass in den Fällen, wo es sich wohl tatsächlich um ein Individuum (und keinen Ort) handelt, z.B. bei Al-lú (Kol. i 8), Flurbezeichnungen mit „festem“ Personennamenelement vorliegen, vergleichbar Bildungen wie aš $\mathrm{a}_{5}-\mathrm{sa}_{6}$ ga-tur (AWEL 31 iii 1), wobei sa ${ }_{6}$-ga als Autonym auch in TSA 10 vii 13 bezeugt ist. Zur Verknüpfung von Flurnamen und Personennamen vgl. jetzt auch Lecompte (2013: 109 Anm. 29).
}

Dr. Thomas E. Balke: SFB 933 „Materiale Textkulturen“, Seminar f. Sprachen u. Kulturen des Vorderen Orients - Assyriologie, Hauptstr. 126, 69117 Heidelberg, Deutschland, E-Mail: thomas.balke@ori.uni-heidelberg.de 
Konstruktion der Ausdrücke mit $\mathrm{du}_{6}$ „Hügel“ + PN bzw. sùr „Senke, Graben“ + PN eher für Lagebeschreibungen denn Autonyme zu sprechen. Das knappe Textformular ohne Einbettung in ein rechtsbezogenes Urkundenformular sprechen ebenso für die Annahme eines Textentwurfes für eine Rechtsurkunde wie auch das abrupte Ende der Urkunde zum Ende der zweiten Kolumne. Nachfolgend sei der kurze Text in Umschrift mit Übersetzung und einem Kommentarteil geboten.

\section{MW 172d (Abb. 1)}

Masse: $\mathrm{H}: 8,9 \mathrm{~cm}$; $\mathrm{Br} .:$ 8,6 cm; D: 1,6 cm;

Datum: ohne Datierung

Vs.

Kol. i

Z.1

8 (bùr) GÁNA

8 (bùr) Feld(fläche)

Z. 2 ĝiš-ùr!-ra

Z. 3 2(bùr) 1 (èše) GÁNA

Z. 4 inim-bànda ${ }^{\mathrm{da}}$ (TUR.DA)

am ĝiš-ùr-ra(-Feld?),

2(bùr), 1(èše) Feld(fläche)

Z. 53 (bùr)1 (èše) GÁNA

des/am? Inimbanda,

Z. $6 \quad \mathrm{du}_{6} \mathrm{sal}_{4}$-la

Z. 7 (bùr) 1 (èše) GÁNA

Z. 8 sùr(LAK 361) al-lú

3(bùr) 1(èše) Feld(fläche)

am (Feld?) ,weiter Hügel“,

3(bùr) 1(èše) Feld(fläche)

an der Senke (des?) Allu;

Kol. ii

Z.1 2(bùr) 1 (èše) GÁNA

Z. $2 \quad \mathrm{du}_{6}$ nam-gi

Z.3 aša ${ }_{5}$ ur!-lugal-gub-kam

Z. 4 ses 5 ní $\hat{g}-b a$

Z. 5 ba- de $_{6}$

Z. 6 aša ${ }_{5}$ ég ní̂̂-ba

Z. 7 gišgal(LAK 648)-si-kam

Z. 8 [ Leerzeile ]

[Die Rückseite der Tafel ist unbeschrieben]

\author{
2(bùr) 1(èše) Feld(fläche) \\ am „Hügel von Namgi“, \\ gehörig zur Flur des UrlugalDU; \\ der Bruder hat die 5 (Parzellen) (als) Geschenk \\ an sich genommen; \\ die „Flur (am) Deich“ ist ein Geschenk \\ des Ĝišgalsi.
}

\section{Kommentar:}

Vs.

i 2: $\quad$ Nach Reinigung eindeutig ĝiš zu Beginn der Zeile, d.h. ĝ iš - ùr-ra zu lesen, das ich allerdings weder als PN noch als FN belegen kann; sowohl ein Zusammenhang mit ĝiš-ùr „Balken, Baumstamm“ als auch mit allerdings erst später belegtem verbalem ĝiš ùr „,eggen“ scheint möglich, vgl. z.B. sargonisch GÁNA giš - ùr (MAD 4, 166: 5) oder häufiges Ur III-zeitliches a-šà ĝiš ùr-ra „Feld, das geeggt worden ist“"(SANTAG 6, 140: 1, 4, 6, ibid. 174: 1, 4, 7; u.ö.). ${ }^{5}$

i 4: Einen Personennamen inim-bànda ${ }^{d a}$ kann ich präsargonisch nicht nachweisen, sondern erst wieder Ur III-zeitlich als inim-tur(-ra) in den Ĝirsu-Urkunden BPOA 1, 115: 5, MVN 22, 175: 11, ibid. 192: 16, ibid. 193 Rs. 6, ibid. 210 Rs. 2 und MVN 13, 10: 4; daher ist m.E. in Analogie zu präsargonischen FN wie aša ${ }_{5}$-gú-bàndada „Feld (am) kleinen Ufer“ (DP 573 i 7) oder aša ${ }_{5}$-ka-gal „Feld 
(an) der großen Kanalöffnung“ (DP 588 ii 7) auch ein Verständnis als ( aš $_{5}$ )-ka-bànda da „(Feld) an der kleinen Kanalöffnung zu überlegen.

i 6: Mit $\mathrm{du}_{6}$ - $\mathrm{sal}_{4}$-la „weiter? Hügel“, das ich sonst nur in TENUS 55, 2 (é $\mathrm{du}_{6}$-sal ${ }_{4}$-la) belegen kann, scheint ein ähnlich wie ki-sal 4 -la gebildeter Ortsname vorzuliegen (s. dazu RGTC 1, 90f.), wobei die semantische (und lautliche!) Differenzierung von sal „fein, dünn, schmal“ (= raqqu) vs. sal $_{4}$ „weit, breit“ (= rapšu) nicht immer klar ist, z.B. im Fall von bur-sal ${ }_{4}$-la „schmale(?) Steinschale“ (AWAS 114 i 3); die exakte semantische Bestimmung einiger sumerischer Dimensionalwörter bereitet, wie ich an anderer Stelle zeigen konnte (Balke 2002), oftmals noch erhebliche Schwierigkeiten, wenngleich hier die Form der Bezugsobjekte für die Zuweisung eines dimensionalen Attributs eine entscheidende Rolle spielt, z.B. was das Antonymenpaar da ĝal „breit“ vs. sig „dünn, eng“ betrifft.

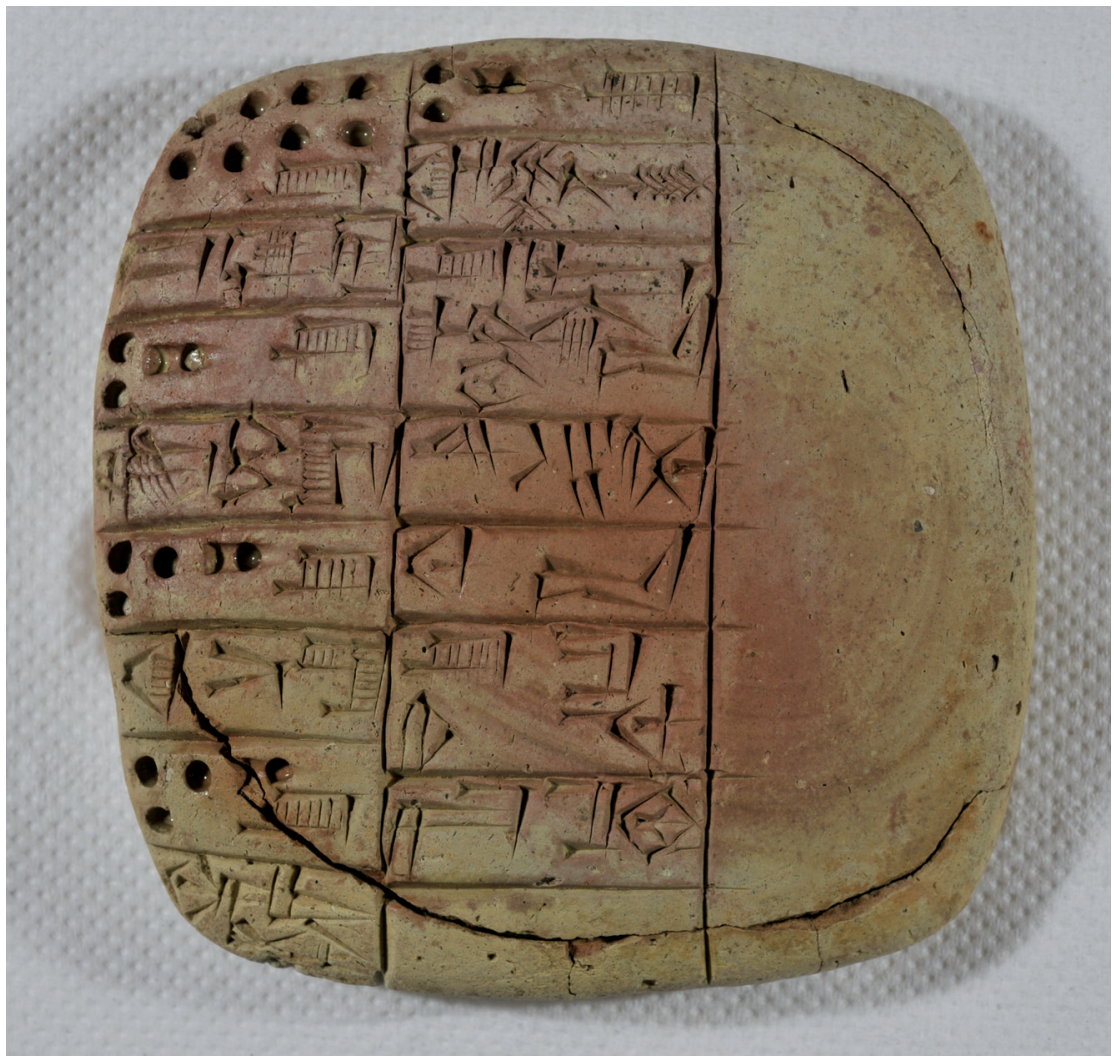

Abb. 1: Fāra-zeitliche Urkunde (MW 172d) des Völkerkundemuseums, Werl.

Geht man nun von einer semantischen Annäherung von $\mathrm{sal}_{4}$ : sal aus, ${ }^{6}$ etwa bei bestimmten Gefäßbezeichnungen, ließe sich hier auch auf alternierendes gur ${ }_{4}$ : gur „dick“ verweisen (s. Balke 2002: 46 mit Anm. 95), auch wenn es sich hierbei m.E. nicht um originär distinkte Wortbasen wie im anderen Fall handelt.

i 8: Nach Kontext ist auch hier eine Lokalisation der vorangehend genannten Parzelle zu erwarten, wonach wohl sùr Al-lú „(Fläche) an der Senke/am Graben des Allu“ zu lesen ist; ein PN Al-lú (< al-lu $u_{5}$ „Krebs“?) etwa ist bezeugt in der sargonischen Urkunde TMTIM 28 iii 6 - im FāraOnomastikon fehlt er -, und ist vielleicht mit den Ur III-zeitlichen Namenformen Al-lu (BPOA 1, 803: 2, ibid. 1216 Rs. 2; BPOA 2, 2006: 5, ibid. 2420: 7; u.ö.) bzw. Al-lu- $\hat{\mathrm{g}} \mathrm{u}_{10}$ (BPOA 7, Rs. 2; MVN 15,

6 Eine andere Form horizontaler Dimensionierung drückt verbales $\mathrm{sal}_{4}$ im Kontext še $\mathrm{sal}_{4}$-la „Gerste, die (zum Trocknen) ausgebreitet ist“"(z.B. ASJ 8, 116 Nr. 32 i 2, 5, 7) aus. 


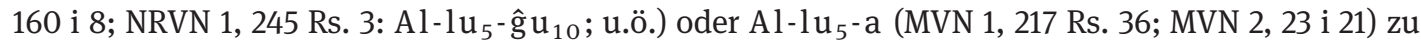
verknüpfen.

ii 2: Ein Toponym $\mathrm{du}_{6}$ nam-gi „Hügel von/des Namgi“ kann ich ansonsten nicht belegen, die Zeichen sind aber nach der Tafelreinigung eindeutig; womöglich sind hier Ortsnamen wie nam-ri $\hat{1}^{\mathrm{ki}}$ (s. RGTC 1, 126) als Vergleich heranzuziehen.

ii 3: Zu diesem PN, bei dem es sich um den bekannten gleichnamigen Kultivator (engar) handeln dürfte, vgl. die Belege bei Andersson (2011: 412f.) (,man of LugalDU') und Pomponio (1987: 255f. s.v. ur-(d) lugal-DU) - zu ergänzen wäre dort FTUM 92 i 2' -, zur Rolle und Funktion des engar zur FāraZeit im allgemeinen vgl. EDATŠ 98-102 mit Table 3; der um sein dimensionales Kasuselement verkürzte PN Ur-lu gal-gub „Hund (i.e. Diener), der [dem] König dient“" ist von seinem Bildungstyp her mit (späteren) Namen wie A-en-ra-gub „Vater, der für den Herrn bereitsteht (d.h. ihm dient)“, dem Namen eines Sohnes IriKAginas zu vergleichen; sowohl Urlugalgub als auch der in Z. 7 genannte Ĝišgal-si erscheinen in der Sammeltafel WF 78 (= EDATŠ 29), Letzterer, ein Viehhirte, auch noch in der Zeugenliste der Hauskaufurkunde WF 30 (= SRU 22 = ELTS 100) ii 3.

ii 4: Ob sich hier ses „Bruder“ auf ein verwandtschaftliches Verhältnis - es müsste sich dann um einen Bruder des Urlugalgub handeln ${ }^{8}$ - bezieht oder ein Kurzname vorliegt ist nicht sicher zu bestimmen, während sich der nachfolgende Numeral „„“ eigentlich nur auf die zuvor genannten 5 Parzellen beziehen kann, die gleichermaßen als nî́̂̆-ba „Geschenk“ bezeichnet sind. Obwohl diese Passage mangels Parallelen m.W. singulär ist, mag man in der knappen Formulierung durchaus einen Hinweis auf eine Erbschaftsangelegenheit sehen.

ii 6-7: Für den PN Ĝišgal-si „,der den Sockel ausfüllt“ s. die Belege bei Pomponio (1987: 155), im präsargonischen Onomastikon von Lagas ist der Name in den Schreibungen Ĝišgal-le-si (ITT 5, 9232 iii 3), Ĝišgal-si (AoF 22, 114 v 7' [engar]; SRU 31 iv 15 [ašgab], ibid. 45 ii 6 [ašgab]; u.ö.) sowie gal Ĝišgal-si (Genava 26, 1 iv 3 [gal-ù ĝ]) bezeugt, während er in den archaischen Texten aus Ur gänzlich fehlt, zum Namenelement s. allerdings Burrows, UET 2, S. 32 Nr. 310-313 u. ibid. AUrSL 308. Es bleibt allerdings in dieser Passage völlig unklar, in welchem (rechtlichen) Zusammenhang die hier beschriebene Schenkung(?) der „Flur (am) Deich“ durch Ĝišgal-si zu dem vorangehenden Sachverhalt steht; die fehlende spezifische Größenangabe dieser Flur mag jedoch darauf hindeuten, dass es sich dabei um eine übergeordnete, größere Flur gehandelt haben mag, in der die einzelnen genannten Parzellen eingebunden waren. Um diese singuläre Urkunde in ihrem Rechtskontext einigermaßen gesichert einordnen zu können, wird man allerdings auf weitere ähnlich strukturierte Textfunde hoffen müssen.

Danksagung: Für die freundliche Aufnahme und Bewirtung beim winterlichen Besuch des Museums Forum der Völker, des Völkerkundemuseums der Franziskaner, in Werl, gilt mein herzlicher Dank P. Reinhard Kellerhoff ofm. Für die im Vorderasiatischen Museum Berlin durchgeführte Reinigung der Tafel sei herzlich J. Marzahn und Fr. Radujkovic gedankt, ebenso wie den Kollegen I. Schrakamp und G. Visicato für erhellende Bemerkungen. Nichtsdestotrotz liegt die Verantwortung für den Inhalt allein bei mir.

7 Bei dem PN Ur-lugal-ra in OSP 1, 1 i 2 könnte es sich um eine Kurzform zu einer Vollform *Ur-lu gal-ra-g ub handeln. 8 Die Urkunde Orient 19 (1983) 2ff. bezeichnet Ur-lug al-gub in Kol. iii 9 als „Bruder des Mannes“ (ses lú), was sich scheinbar auf den in Kol. iii 1 genannten Verkäufer namens nam - ma ḩ bezieht. 


\section{Literatur}

Andersson, J. (2011): Kingship in the Early Mesopotamian Onomasticon 2800-2200 BCE (Studia Semitica Upsaliensia 28). Uppsala.

Balke, Th.E. (2002): Die sumerischen Dimensionaladjektive nim und sig. Anmerkungen zur Polysemie und Grammatikalisation dimensionaler Ausdrücke im Sumerischen, in: O. Loretz et al. (Hrg.), Ex Mesopotamia et Syria Lux. Festschrift für Manfried Dietrich zu seinem 65. Geburtstag (AOAT 281), Münster, 31-53.

Burrows, E. (1935): Archaic Texts (UET 2), London.

Krebernik, M. (1998): Die Texte aus Fāra und Tell Abū Șalābīḥ, in: P. Attinger / M. Wäfler (Hrg.), Mesopotamien. Späturuk-Zeit und Frühdynastische Zeit. Annäherungen 1 (OBO 160/1), Fribourg/Göttingen, 237-427.

Lecompte, C. (2013): Zu Dörfern, Weilern und ländlichen Siedlungen in vorsargonischer Zeit: Die Entwicklung der Bezeichnungen $E_{2}$ und $E_{2}-D_{U R U}$, RSO 86,103-120.

Martin, H.P. et al. (2001): The Fara Tablets in the University of Pennsylvania Museum of Anthropology, Bethesda, MA.

Ozaki, T. (2008): Three Early Dynastic Sumerian Sale Contracts of Immovables Housed in the Okayama Orient Museum, JAC 23, 55-61.

Pomponio, F. (1987): La prosopografia dei testi presargonici di Fara (StSem. NS 3), Rom.

Pomponio, F. / G. Visicato (1994): Early Dynastic Administrative Tablets of Šuruppak (IUO Ser. Maior $6=$ MEE 6), Neapel. Wilcke, Cl. (2007): Early Ancient Near Eastern Law: A History of its Beginnings. The Early Dynastic and Sargonic Periods, 2. erweiterte Aufl., Winona Lake.

Hinweis: Abkürzungen nach W. von Soden, Akkadisches Handwörterbuch, Wiesbaden 1959-1981 (AHw) sowie ergänzend OrNS 63 (1994) $1^{\star}-3^{*}$ und Reallexikon der Assyriologie und Vorderasiatischen Archäologie (RIA). Weitergehende Abkürzungen sind: AUrSL = Burrows, UET 2 (1935) Sign List: pl. 1-34; EDATŠ = Pomponio / Visicato (1994); FTUM = Martin et al. (2001); TMTIM = P. Steinkeller / J.N. Postgate, Third-Millennium Legal and Administrative Texts in the Iraq Museum, Baghdad (MesCiv 4, 1992); ELTS = I.J. Gelb / P. Steinkeller / R.M Whiting, Jr., Earliest Land Tenure Systems in the Near East: Ancient Kudurrus (OIP 104, 1989-1991). 